Lucrările Seminarului Geografic Dimitrie Cantemir

Vol. 44, October 2017, pp. 109-124

http://dx.doi.org/10.15551/lsgdc.v44i0.08

\title{
Impacts de la variabilité climatique et de la pression démographique sur les ressources en eau du bassin de Thysse Kaymore (Saloum-Sénégal)
}

\author{
Waly Faye ${ }^{1}$, Didier Orange ${ }^{2}$, Alioune Kane ${ }^{1}$, Awa Fall Niang ${ }^{1}$, \\ Ionuț Minea ${ }^{3}$
}

${ }^{1}$ Université Cheikh Anta Diop de Dakar, Sénégal

2 IRD, UMR 210, Eco\&Sols, Montpellier, France

${ }^{3}$ Alexandru Ioan Cuza University of Iasi, Romania

To cite this article: Faye, W., Orange, D., Kane, A., Niang, A.F., Minea, I. (2017). Impacts de la variabilité climatique et de la pression démographique sur les ressources en eau du bassin de Thysse Kaymore (Saloum-Sénégal). Lucrările Seminarului Geografic Dimitrie Cantemir, Vol. 44, pp. 101-108. DOI: $10.15551 / 1$ gdc.v44i0.08

To link to this article: http://dx.doi.org/10.15551/lsgdc.v44i0.08 


\title{
IMPACTS DE LA VARIABILITÉ CLIMATIQUE ET DE LA PRESSION DÉMOGRAPHIQUE SUR LES RESSOURCES EN EAU DU BASSIN DE THYSSE KAYMORE (SALOUM-SENEGAL)
}

\author{
Waly Faye ${ }^{1}$, Didier Orange ${ }^{2}$, Alioune Kane ${ }^{3}$, \\ Awa Fall Niang ${ }^{4}$, Ionuț Minea ${ }^{5}$
}

\begin{abstract}
Résumé. Dans le bassin arachidier (en zone soudano-sahélienne) et particulièrement dans le bassin de Thysse Kaymore, le déficit pluviométrique continu et prononcé depuis les années 1970 et la pression démographique croissant ont conduit à la dégradation des ressources en eau, rendant de plus en plus difficile l'accès à l'eau. Les eaux souterraines sont souvent très profondes, alors que les eaux de surface qui devraient servir de recours dans de pareilles situations connaissent un tarissement précoce. Dans ce petit bassin de $162 \mathrm{~km}^{2}$, le déboisement massif, la mise en culture de zones marginales et la disparition de la jachère conjugués au contexte climatique défavorable et à la pression démographique ont entrainé la dégradation du réseau hydrographiques et modification du fonctionnement hydrologique. Pour étudier les impacts de la péjoration climatique et de la pression anthropique sur les ressources en eau du bassin de Thysse Kaymore et sur les conditions de vie des populations, nous avons effectué l'analyse des séries chronologiques des précipitations de 1918 à 2011, des mesures piézométriques ainsi que des débits. Pour l'analyse diachronique de l'évolution des espaces ruraux des images Landsat de 1986, 1996, 2007 et 2016 sont utilisés. Les déficits pluviométriques annuels varient entre 54 et $60 \%$. On constate un abaissement du niveau des nappes phréatiques, le tarissement précoce des puits, l'assèchement et l'ensablement des bas-fonds, une profondeur importante du niveau de l'eau sous le sol $(58$ à $116 \mathrm{~m}$ pour le Continental Terminal et $300 \mathrm{~m}$ pour le Maestrichtien) dans cette zone rurale ou les eaux souterraines sont les principales sources d'approvisionnement en eau des populations. A cette péjoration pluviométrique, il faut ajouter la forte pression démographique avec l'augmentation de la population qui a doublé en 30 ans (de 9400 habitants en 1976 elle est passée à 22524 en 2013) entrainant la multiplication des besoins en eau. Nonobstant ces problèmes accrus d'approvisionnement en eau potable des populations, cette zone constitue un bassin agricole, dont $62 \%$ des $90,76 \%$ de sa superficie cultivable sont exploités. Dans le même temps, on note le développement du maraîchage, une activité fortement consommatrice d'eau. Les besoins futurs en eau seront donc en croissance. Il
\end{abstract}

\footnotetext{
${ }^{1}$ Université Cheikh Anta Diop de Dakar, Faculté des Lettres et des Sciences Humaines, Département de Géographie, walyjunior@hotmail.fr

${ }^{2}$ IRD, UMR 210, Eco\&Sols, Montpellier, France, Didier.orange@ird.fr

${ }^{3}$ Université Cheikh Anta Diop de Dakar, Faculté des Lettres et des Sciences Humaines, Département de Géographie, alioune.kane@ucad.edu.sn

${ }^{4}$ Université Cheikh Anta Diop de Dakar, Faculté des Lettres et des Sciences Humaines, Département de Géographie, n_awa@hotmail.com

${ }^{5}$ Alexandru Ioan Cuza University of Iasi, Faculty of Geography and Geology, Department of Geography, ionutminea1979@yahoo.com
} 
est donc important de considérer un modèle durable de l'alimentation correcte en eau de cette population rurale.

Mots clés : variabilité climatique, ressources en eau, bassin versant, bassin agricole

\section{Introduction}

Depuis quelques décennies, on assiste à une dégradation poussée de la situation écologique du bassin arachidier, et particulièrement dans la partie sud-est. L'évolution des écosystèmes dans cette zone, notamment à Thysse Kaymore dans les années 80 a préoccupé des institutions comme l'IRD, le CIRAD, l'ISRA qui ont mené des programmes pour la gestion des ressources naturelles de cette région. Ainsi, de 1983 à 1996, le bassin de Thysse Kaymore a abrité une station d'expérimentation de l'IRD dans le cadre d'un programme de recherche pluridisciplinaire sur le fonctionnement hydrologique et l'aménagement hydroagricole des bassins versants expérimentaux de Thysse Kaymore.

En effet, la détérioration des paysages de ce bassin intervient dans un contexte pédoclimatique et socio-économique défavorable marqué par une baisse chronique et une forte variabilité interannuelle de la pluviométrie. Les écoulements des cours d'eau dans cette région suivant les tendances pluviométriques et s'organisant en fonction de l'alimentation reçu depuis les bassins versants, la baisse pluviométrique a provoqué le tarissement de beaucoup de cours d'eau. Le déficit pluviométrique qui s'est amorcé dans le Sahel en 1968 (Dacosta et al., 2002 ; Descroix et al., 2009 ; Mahé et al., 2013), s'est accompagné d'un dessèchement de la zone soudano-sahélienne qui s'est traduit par un épuisement des eaux superficielles, ce qui a été confirmé dans la région du Sine-Saloum par les études de Lienou et al. (1999). A cette vulnérabilité hydrologique s'ajoute la forte pression démographique ainsi que la mécanisation et la vulgarisation agricole qui ont eu comme corollaire un déboisement massif, un épuisement des terres et la disparition de la jachère.

La région de Thysse Kaymore se trouve dans le bassin arachidier, une zone agroécologique d'importance capitale qui concentre près de $60 \%$ de la population rurale et fournit l'essentielle de la production agricole du pays (ISRA, 2010). Il représente $57 \%$ des 3,8 millions d'hectares des terres arables du pays (DAPS, 2009) et fourni $2 / 3$ de la production d'arachide et de mil du Sénégal (GCO/SEN, 2007). Toutefois, l'importance de cette région comme l'ont souligné beaucoup d'auteurs (Albergel et al. 1989 ; Diatta, 1994 ; Lericolais 1972, 1999 ; Perez 1994 etc.) est menacée depuis quelques années par la dégradation du milieu qui résulte de trois facteurs principaux : les aléas climatiques, la baisse de la fertilité des sols en culture continue, et l'érosion hydrique qui sévit particulièrement dans le Sud-est, région de Thysse Kaymore. Cette région enregistre en années décennale humide $1000 \mathrm{~mm}$ et en année décennale sèche 500mm (Diatta, 1994). La moyenne annuelle des précipitations sur la période de 1930-1990 qui était de $820 \mathrm{~mm}$ est passé à $664 \mathrm{~mm}$ pour la période de 1970-1990 (Albergel et al.1995). Cette forte réduction de la pluviométrie intervient dans le contexte d'une anthropisation sans précédent du bassin. Selon Perez (1994), les surfaces correspondantes aux parcours, jachères, culture représentaient respectivement 62\%, 10\%, 28\% de la superficie totale du Sud du Saloum en 1970 alors qu'en 1983 on obtient $34 \%$ de forêts, $2 \%$ de jachères et $64 \%$ de cultures, soit un plus que doublement des surfaces cultivées en 13ans. Ces espaces cultivés qui occupaient $31 \%$ de la surface du bassin de Thysse en 1986 sont passé à 62\% en 2016. Cette dégradation des états 
de surface fait que l'érosion hydrique entraine des pertes en terres proche de 1 tonne/ha/an (Albergel et al. 1989).

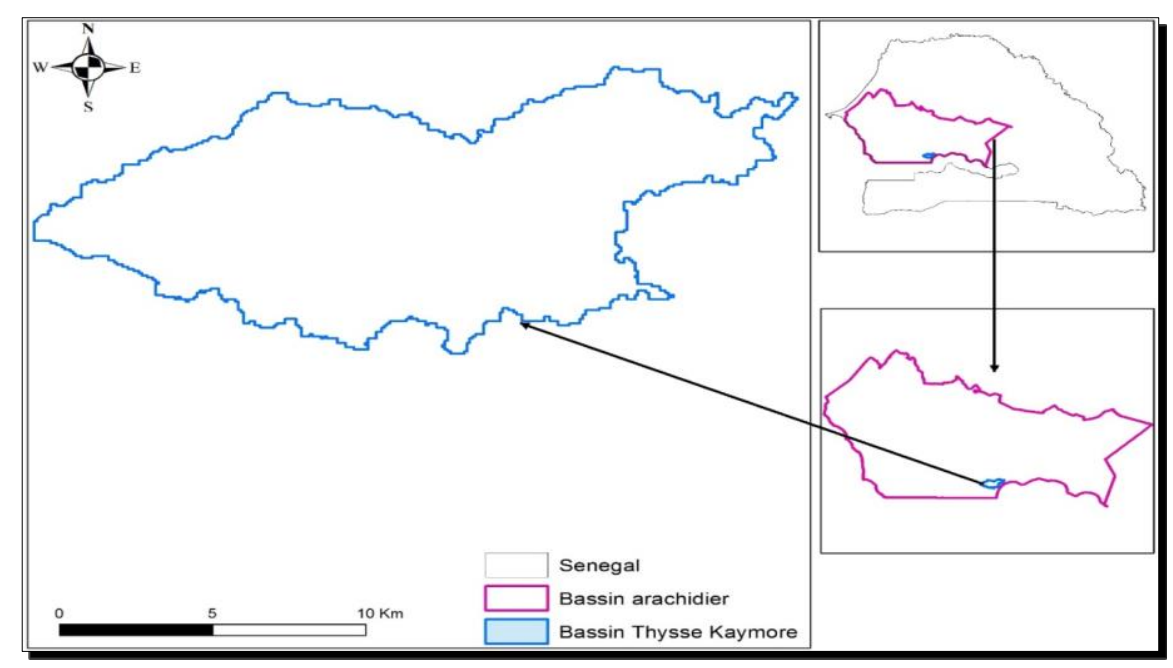

Figure 1 : Localisation du bassin versant de Thysse Kaymore

Ces pertes en terres cultivables combinées à l'augmentation démographique et à la mécanisation de l'agriculture ont entrainé une intensification de l'utilisation des terres avec la réduction, voire la disparition de la jachère et l'extension des cultures à des zones fragiles. Ainsi, le manque de terres agricoles, la pauvreté et la dégradation des sols, la sécheresse, le recul des cultures sous pluie ont entrainé une mutation des systèmes agraires. Il s'en n'est suivi un défrichement massif, la mise en culture des bas-fonds et une accélération du ruissellement. Dans cette zone, la modification des états de surface des sols fait que malgré la baisse pluviométrique, le ruissellement dans le bassin de Thysse Kaymore est très agressif. Cette intensification du ruissellement entraine la dégradation du réseau hydrographique avec l'ensablement des bas-fonds, mais aussi la modification du fonctionnement hydrologique avec la réduction de la fraction infiltrée.

Cette modification des conditions de transfert et de stockage des eaux entraine la baisse du niveau des nappes, le tarissement précoce des eaux de surfaces et de certains puits. Ce qui pose un problème de disponibilité en eau pour les populations car les eaux souterraines sont les uniques ressources en eau disponibles pendant une bonne partie de l'année.

\section{Matériels et méthode}

L'analyse de l'impact de la péjoration climatique et des actions anthropiques sur les ressources en eau du bassin de Thysse Kaymore à nécessité quatre méthodes d'investigation : l'analyse des données hydropluviométriques, l'analyse des données démographiques, l'analyse des données piézométriques, et le traitement et l'analyse d'images satellitaires. 


\subsection{Les données hydropluviométriques}

\subsubsection{Les données pluviométriques}

Ce sont les données mensuelles de la station synoptique de Kaolack et des postes pluviométriques de Kaffrine, de Nioro et de Thysse Kaymore. Ces données sont recueillies au niveau de l'Agence National de l'Aviation Civile et de la Météorologie (ANACIM) pour la période de 1970-2013, du recueil de pluviométrie de l'ORSTOM (actuelle IRD) pour la période de 1918 à 1969 ainsi que des rapports de campagne sur la station expérimentale de Thysse. Ces données ont été homogénéisées suivant la méthode des doubles cumuls, appliquée aux stations appartenant au même domaine climatique. Cette méthode s'appuie sur la comparaison de la période d'observation commune aux deux stations $\mathrm{A}$ et $\mathrm{B}$ en se fondant sur l'hypothèse que la station $A$ est homogène et la station $B$ hétérogène. C'est le cumul des totaux annuels des stations jusqu'à un temps (ti), (i) étant la période commune aux deux stations en partant de l'année la plus récente à l'année la plus ancienne. $\mathrm{Si}_{\mathrm{A}}$ (i) correspond au double cumul de la station $\mathrm{A}$ et $\mathrm{T}_{\mathrm{B}}$ (i) au cumul de la station $\mathrm{B}$ :

$$
\begin{aligned}
& \mathrm{T}_{\mathrm{A}}(\mathrm{i})=\mathrm{P}_{\mathrm{A}}(1)+\mathrm{P}_{\mathrm{A}}(2)+\ldots+\mathrm{P}_{\mathrm{A}}(\mathrm{i}) \\
& \mathrm{T}_{\mathrm{B}}(\mathrm{i})=\mathrm{P}_{\mathrm{B}}(1)+\mathrm{P}_{\mathrm{B}}(2)+\ldots+\mathrm{P}_{\mathrm{B}}(\mathrm{i})
\end{aligned}
$$

Si les données sont homogènes les points sont alignés sur une droite (pente de la droite : $\left.\mathrm{P}_{\mathrm{Bmoy}} / \mathrm{P}_{\mathrm{Amoy}}\right)$. Par contre si les données de la station $\mathrm{B}$ sont hétérogènes on observe une cassure dans l'alignement des points. En cas de rupture, la correction de la station lacunaire est effectuée en calculant le coefficient de correction obtenu par le rapport de $\mathrm{m} / \mathrm{m}$ des stations $\mathrm{A}$ et $\mathrm{B}$, et qui est multiplié par chacun des totaux annuels lacunaires en commençant par l'année qui marque la cassure : m étant la moyenne de la première période et $\mathrm{m}$ ' la moyenne de la deuxième période. L'estimation d'une valeur annuelle de la station incomplète s'obtient par l'application de la formule de Roche, (1963)'

$$
y x=\bar{y} k+r x y * \frac{\sigma y x}{\sigma x k}(x-\bar{x} k)
$$

$\bar{y} k=$ moyenne de la station incomplète,

$x=$ valeur de la station de référence à une année $\mathrm{x}$,

$\bar{x} k=$ moyenne de la station de référence,

$\mathrm{k}=$ le nombre d'années communes entre les deux stations

L'indice d'aridité de Martonne (1923) est utilisé pour évaluer la tendance climatique de la région. C'est le rapport entre la hauteur moyenne des précipitations annuelles et la moyenne des températures annuelles: $\mathrm{i} a=\mathrm{P} /\left(\mathrm{t}^{\circ}+10\right)$ (Minea, Stanga, 2004).

\begin{tabular}{|c|c|}
\hline Valeur de I & Type de climat \\
\hline 0 à 5 & Hyper aride \\
\hline 5 à 10 & Aride \\
\hline 10 à 20 & Semi-aride \\
\hline 20 à 30 & Semi humide \\
\hline 30 à 40 & Humide \\
\hline 40 à 55 & Humide \\
\hline
\end{tabular}

\footnotetext{
${ }^{6}$ M. Roche, (1963) : Hydrologie de surface, GAUTHIER- VILLARS ÉDITEUR - PARIS 1963
} 


\subsubsection{Les données hydrologiques}

Ce sont les débits journaliers de la station de Thysse de 1983 à 1992 fournies par la DGPRE. Les débits annuels sont calculés et mis en relation avec la pluviométrie pour analyser la relation entre l'évolution pluviométrique et le ruissellement sur le bassin versant et la variation des débits.

\subsection{Les données démographiques}

Elles concernent les données des 25 villages qui se trouvent dans le bassin versant de Thysse Kaymore pour les recensements de 1988 et 2002 et 2013. Ces données sont recueillies au niveau de l'Agence Nationale des Statistique et de la Démographie (ANSD). Le traitement statistique de ces données a permis d'évaluer le degré d'anthropisation du bassin de Thysse Kaymore de 1988 à 2013.

\subsection{Les données piézométriques et de forage}

Cette analyse s'est basée sur les données fournies par la DGPRE sur l'ensemble des forages qui sont forés dans le bassin de Thysse Kaymore en ce qui concerne les nappes captées, les profondeurs des forages, les rabattements, les débits et les paramètres physicochimiques. Nous avons ajouté à ces données les mesures piézométriques de quatre puits qui ont été suivis régulièrement pendant la période d'étude du projet pilote Sine-Saloum basfonds de Thysse Kaymore. Une série de mesures sur l'ensemble du dispositif, réalisée en juillet, octobre1989 et février 1990 dans le cadre de ce programme. Ces données sont analysées en relation avec l'évolution des états de surface, la réduction des précipitations et l'augmentation démographique du bassin de Thysse.

\subsection{Les données satellitaires utilisées}

Des images satellitaires Landsat sont utilisées pour l'étude diachronique de l'estuaire du Saloum. Ce sont les images Landsat de 1986, 1996, 2007 et 2016 téléchargées à partir de GLOVIS. Ces images ont fait l'objet d'une composition colorée suivie d'une classification supervisée à l'aide de EVI 4.7 pour déterminer la dynamique des paysages. Elle est effectuée selon le principe des compositions colorées qui consiste à affecter aux trois couleurs primaires (rouge, vert, bleu) trois images acquises au-dessus d'une même région, au même moment mais dans des longueurs d'onde différentes. On a utilisé la combinaison des bandes 453 qui est une composition nette qui permet une identification claire des différents types de végétation et l'interface terre-eau est aussi est très claire. Les unités du paysages prises en compte dans la classification sont au nombre de 5 classes : eau, savane arbustive, savane arborée, sols cultivés. On a extrait des signatures spectrales et on a réalisé la classification totale de l'image sur la base des signatures spectrales obtenues. Les résultats sont cartographiés pour visualiser l'évolution des paysages. 


\section{Résultats et discussion}

\subsection{L’évolution pluviométrique dans le bassin de Thysee}

Le bassin de Thysse Kaymore qui couvre environ $162 \mathrm{~km}^{2}$ à l'extrême sud-est du bassin arachidier et à proximité du baobolong qui se jette dans la Gambie est sous la dépendance du climat soudano-sahélien et enregistre en année décennale humide $1000 \mathrm{~mm}$ et en année décennale sèche des précipitations de $550 \mathrm{~mm}$ (Diatta, 1994). Mais depuis les années 70, la sécheresse qui frappe le Sahel et qui a entrainé la migration des isohyètes vers le sud du pays a fortement éprouvé la région. Ainsi, la moyenne annuelle des précipitations sur la période de 1930-1990 qui était de $820 \mathrm{~mm}$ est passé à $664 \mathrm{~mm}$ pour la période de 1970 1990 (Albergel et al.1995). L'étude de l'évolution spatiotemporelle des précipitations à partir des stations de Kaolack, Nioro et Kaffrine qui sont les plus complètes de la région montre une forte réduction de la pluviométrie avec une alternance de périodes humides et de périodes sèches.

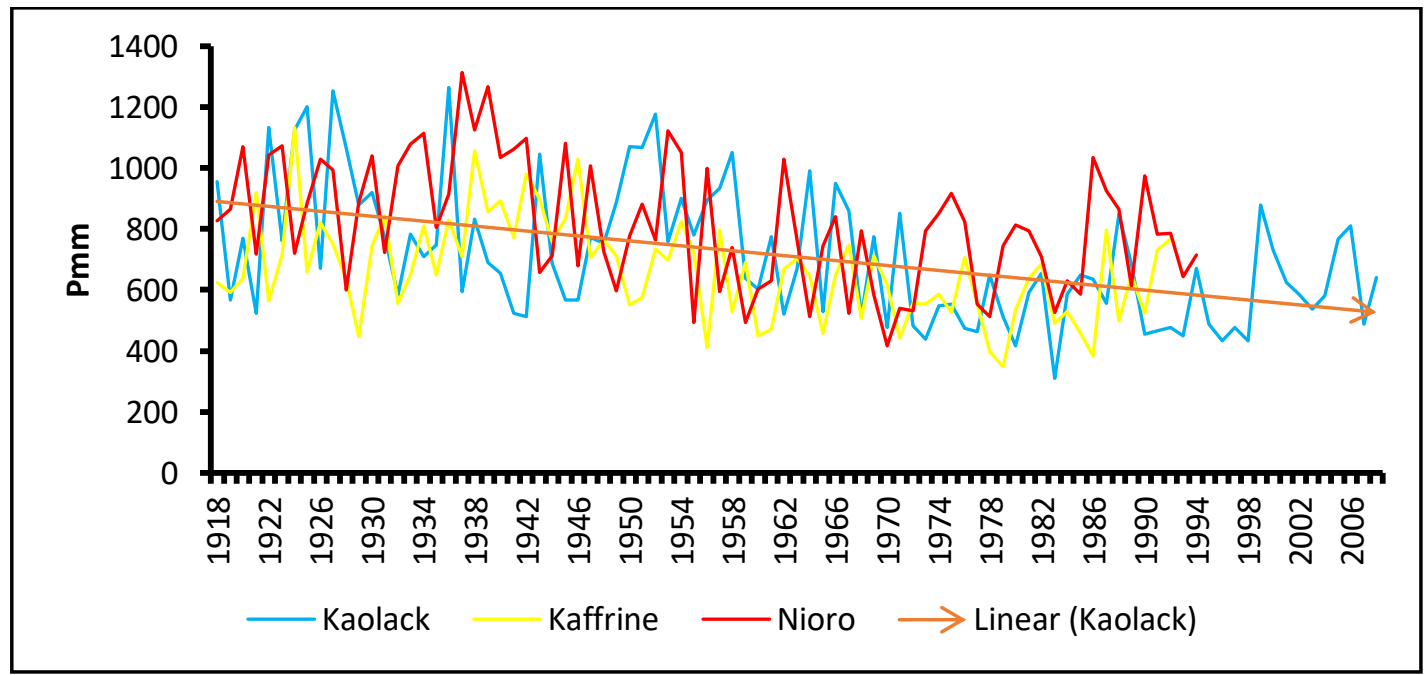

Figure 2 : L'évolution pluviométrique de la région

L'évolution pluviométrique montre une alternance de périodes humides et de périodes sèches, dont la plus longue et la plus drastique est celle des années 70 avec des déficits pouvant atteindre 397mm à Nioro en 1983, 319mm à Kaffrine en 1984 et 400mm en 1983 à Kaolack. L'évolution pluviométrique de la région montre une tendance globale à la baisse des totaux pluviométriques annuels au niveau de toutes les stations. Cette baisse pluviométrique marquée par une migration des isohyètes vers le sud ne met pas seulement en évidence la réduction des totaux pluviométriques annuelles, mais surtout la forte variabilité annuelle et interannuelle de la pluviométrie. 


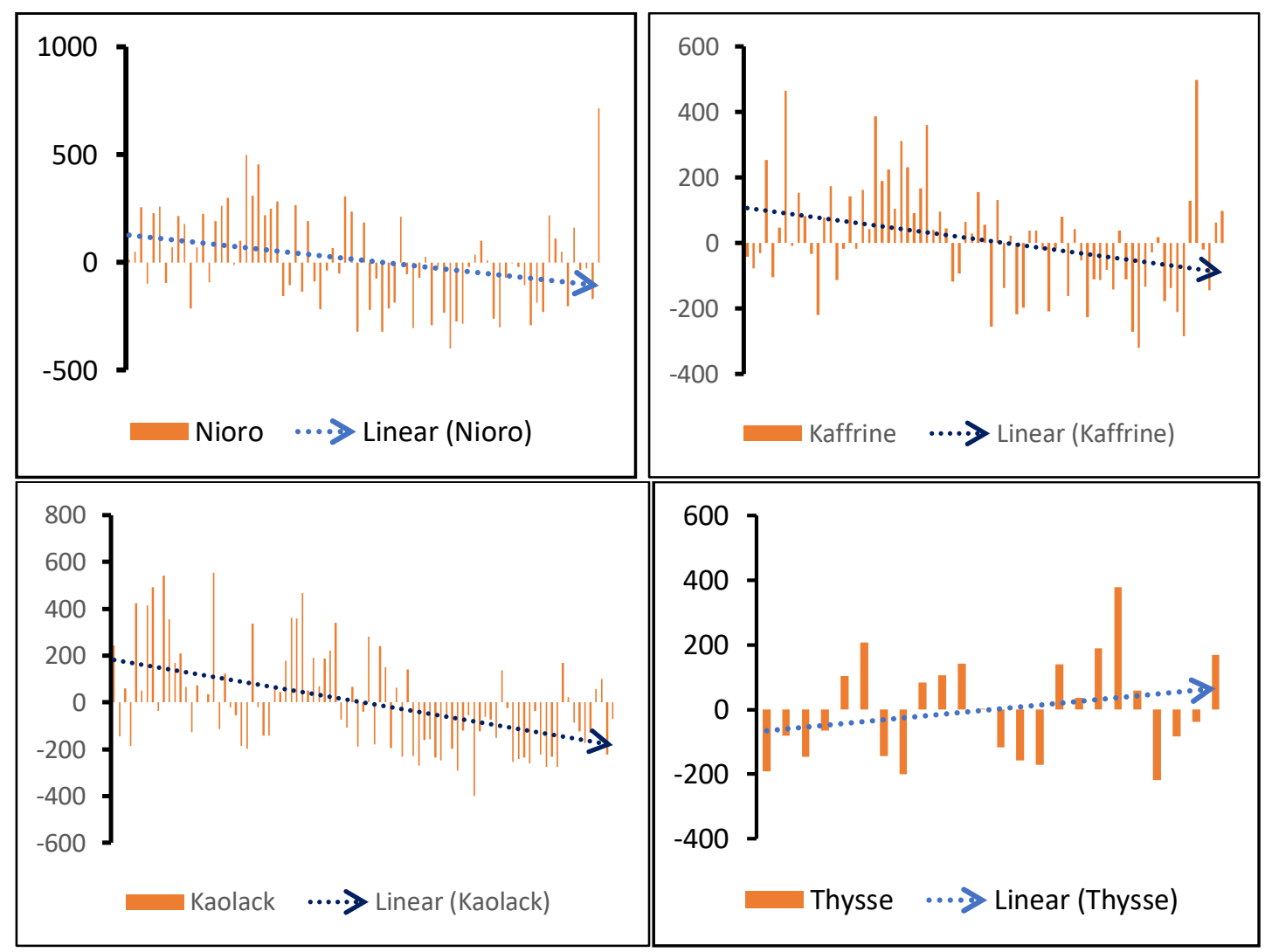

Figure 3 : Variation interannuelle de la pluviométrique

La décroissance des totaux pluviométriques annuels s'est accompagnée d'une forte variabilité interannuelle des précipitations, accentuant l'intensité de la sécheresse. Cette baisse des précipitations constitue selon Sagna $(1995)^{7}$ un signal important des modifications climatiques qui ne concernent pas que le Sahel. Cette tendance générale à la baisse sur le Sahel est due à l'évolution temporelle même de la pluviométrie marquée par une persistance de la sécheresse ${ }^{8}$. La situation de la station de Thysse qui montre une tendance à la hausse est due au fait que la série obtenue se trouve dans la période de sécheresse et se termine par une petite phase humide. La fluctuation des totaux annuelles des précipitations est caractéristique des précipitations dans la zone Soudano-Sahélienne (Dacosta et al. 2002). Ces fluctuations se manifestent à la fois dans le temps et dans l'espace, et s'exprime tant sur les précipitations annuelles que sur la hauteur des pluies journalières. L'indice d'aridité de Martonne qui varie entre 10 et 20 pour la période de 1981 à la fin des séries est caractéristique d'un climat semiaride et montre une tendance à l'aridité. Pour la station de Thysse, de 1970 à 1993, seul les années 1987 et 1988 sont humides avec des indices respectifs de 21,3 et 26,3 pour une pluie

\footnotetext{
${ }^{7}$ Sagna P. (1995) : L'évolution pluviométrique récente de la Grande-Côte du Sénégal et de l'archipel du Cap-Vert, article, Revue de géographie de Lyon, Année 1995, Volume 70, Numéro 3, pp. 187-192 ${ }^{8}$ Diallo M. A. (1995) : Evolution de la pluviométrie au Sahel, Internal Repport, Inetrnational Atomic Energy Agency and United Nations Educational Scientific and cultural organisation, Inetrnational centre for theorical physic, $6 \mathrm{p}$.
} 
respective de 840 et $1029 \mathrm{~mm}$. Cette réduction des apports pluviométriques entraine le tarissement précoce des ressources en eau de surface et une forte sollicitation des eaux souterraines pour la satisfaction des différents besoins en eau de plus en plus croissants.

\subsection{L'anthropisation et ses impacts sur le bassin de Thysse kaymore}

Malgré la sécheresse persistante, la population du bassin versant de Thysse Kaymore qui s'active majoritairement dans l'agriculture et l'élevage a fortement augmenté au cours des dernières décennies.

En dépit de la sécheresse, la population du bassin de Thysse kaymore a fortement augmenté. Elle a doublé entre 1988 et 2013 en passant de 10679 à 23140 hbts. Cette augmentation fulgurante de la population dans une zone agricole est allée crescendo avec l'accroissement de la demande en terre à cultiver. Il s'en est suivi un déboisement massif, une surexploitation des terres, la disparition de la jachère, l'exploitation de zones marginales comme les bas-fonds et les versants. Ainsi, souligne Perez (1994), les surfaces correspondantes aux parcours, jachères, culture représentaient respectivement $62 \%, 10 \%$, $28 \%$ de la superficie totale du Sud du Saloum en 1970 alors qu'en 1983 on obtient 34\% de forêts, $2 \%$ de jachères et $64 \%$ de cultures, soit un plus que doublement des surfaces cultivées en 13ans. Cette situation témoigne de l'intense anthropisation du bassin versant dont les sols sont de plus en plus exposés aux différentes formes d'érosion, particulièrement hydrique.

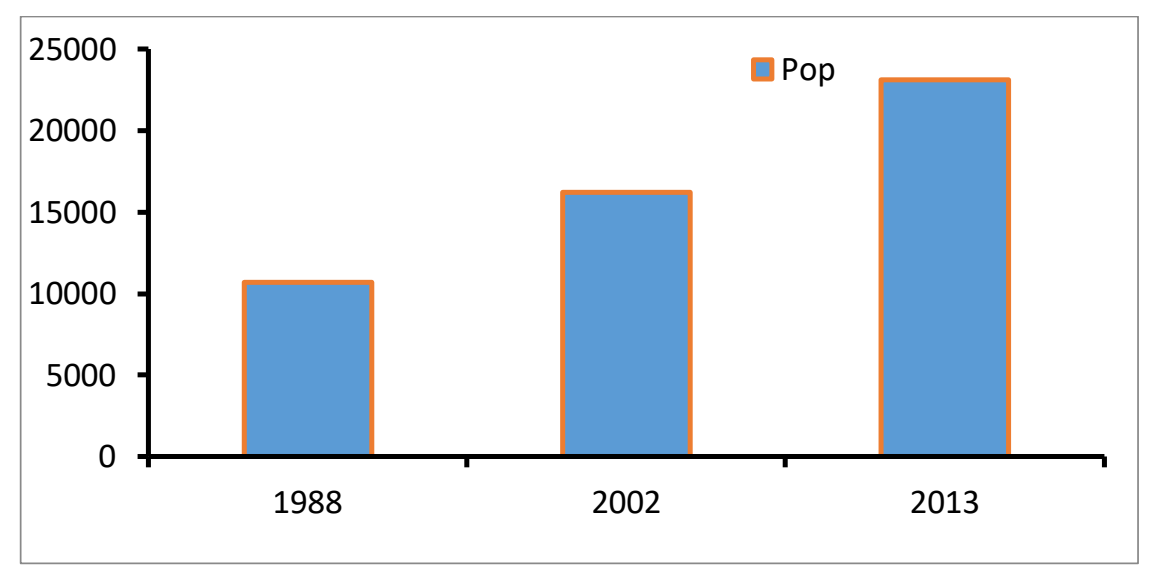

Figure 4: Evolution de la population du bassin versant de Thysse

Cette anthropisation croissante conjuguée à la péjoration climatique a entrainé une forte dégradation des paysages et la modification des états de surfaces du bassin versant avec des conséquences fortes sur le fonctionnement hydrologique de ce dernier.

L'analyse des images satellitaires Landsat de 1986, 1996, 2007 et 2016 permet de mettre en évidence l'évolution des paysages du bassin versant de Thysse Kaymore pendant les années de début de la sécheresse, durant la sécheresse et pendant la période de retour pluviométrique constatée depuis le début des années 2000. 

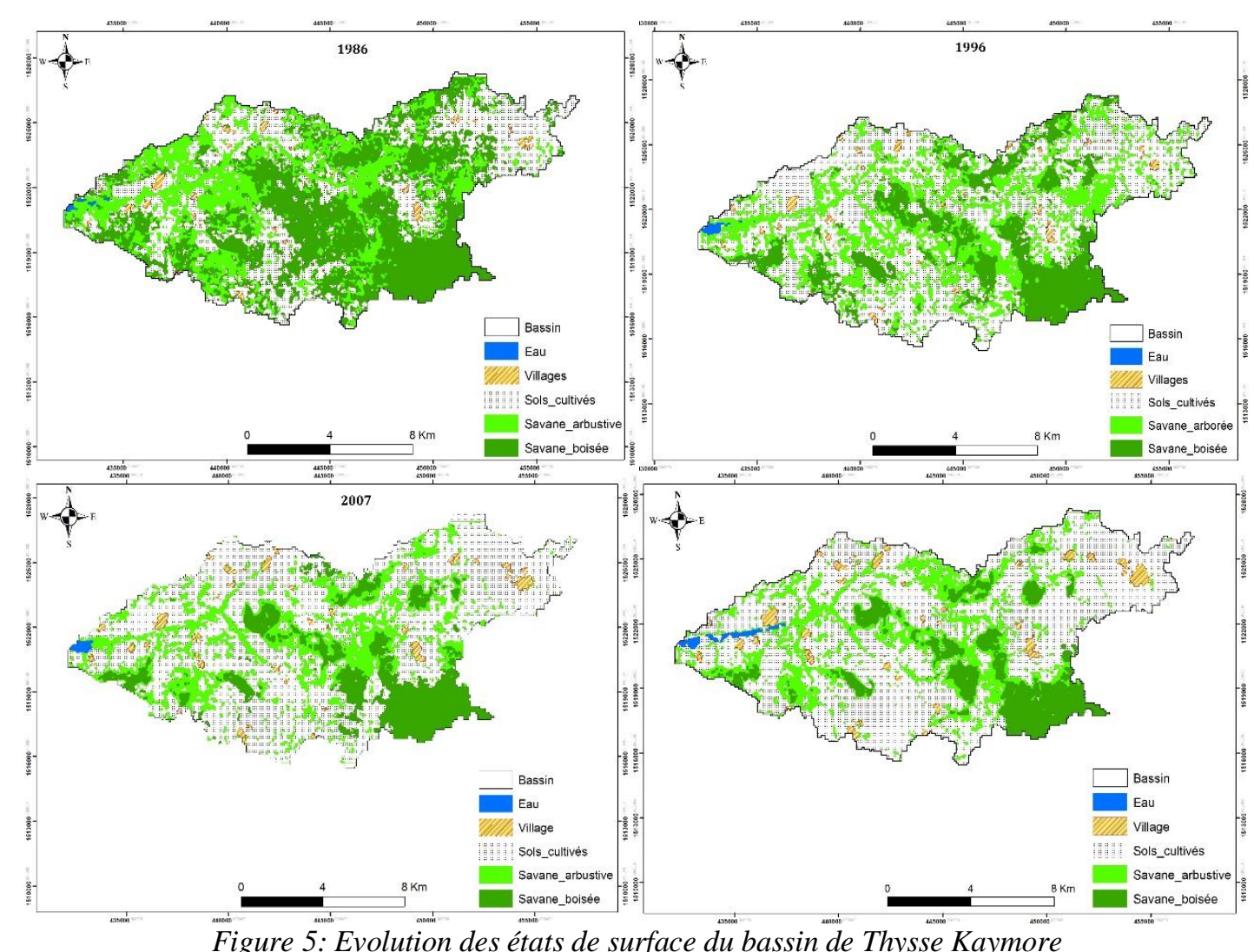

L'effet déterminant est la pression exercée sur les ressources naturelles avec notamment l'intensification de l'utilisation des sols mise en évidence par la forte extension des zones de culture au détriment des espaces boisés. Les sols cultivés qui ne représentaient que $31 \%$ de la surface du bassin sont passés à $62 \%$ aujourd'hui, soit un doublement des espaces cultivés entre 1986 et 2016. Consécutivement à l'extension des espaces cultivés, les savanes arbustives et boisées qui représentaient respectivement en $198627 \%$ et $40 \%$ occupent en $201621 \%$ et $12 \%$. Les savanes boisées qui occupaient l'essentiel du bassin en 1986 constituent aujourd'hui le paysage le moins représentatif du bassin. D'ailleurs, les 12\% qui subsistent encore de ce type de paysage le sont grâce à la forêt classée de Ngayene qui occupe une portion sud-est du bassin. La forêt classée de Ngayene créee en 1950, couvrent actuellement 1900 ha dont les $43 \%$ se trouvent dans le bassin versant de Thysse. Ces $43 \%$ représentent 39\% des savanes boisées du bassin versant de Thysse Kaymore. Cette forte dégradation des états de surface augmente le ruissellement, le déficit d'infiltration et l'accélération de l'érosion très active dans la région. Selon Ndong (1995) l'infiltration dépend des conditions de surface du sol et des caractéristiques d'intensités/durée de pluie.

Et l'un des effets de la sécheresse est de réduire la protection du sol, d'augmenter le ruissellement et donc de diminuer l'infiltration et le stock d'eau utile pour les plantes. Ce qui fait que dans ce bassin, malgré la faiblesse de la topographie et la péjoration pluviométrique, les ruissellements sont parfois violents, provoquant d'important ravinement. Ainsi, la modification des états de surface a fortement contribué à la dégradation des ressources en 
eau du bassin avec notamment l'ensablement progressif des bas-fonds qui entraine la désorganisation des conditions de stockage et de transfert des eaux.

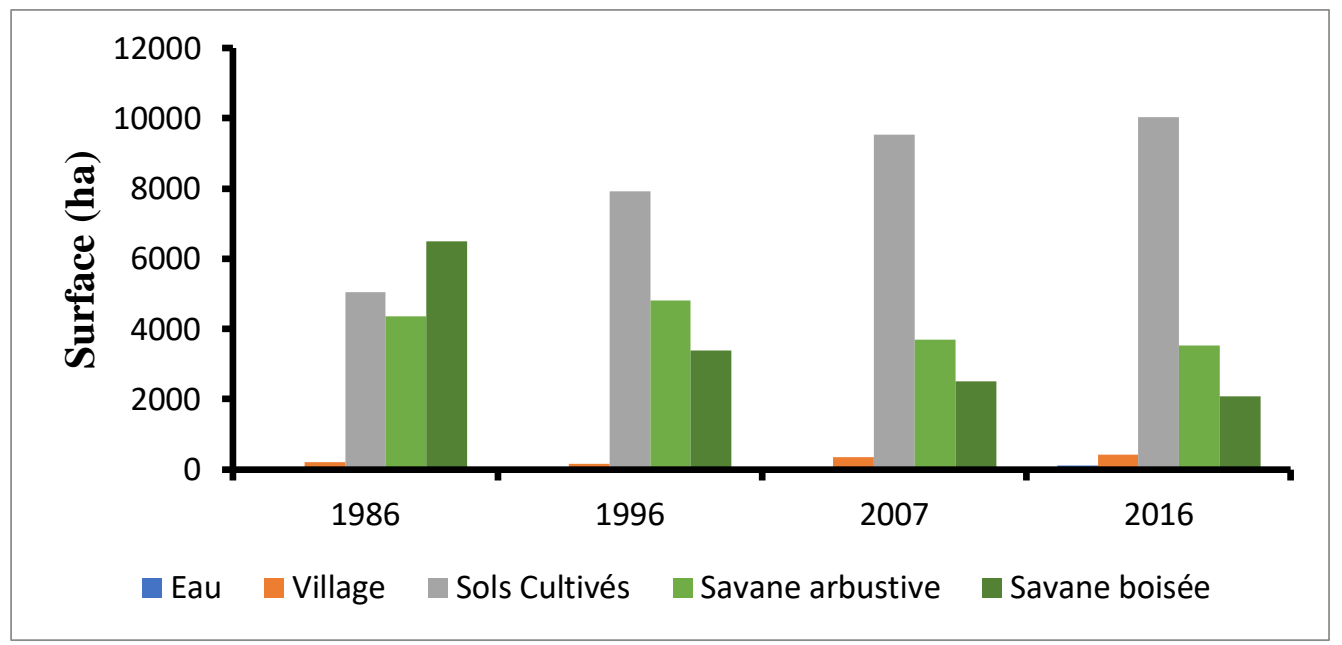

Figure 6: Evolution des différents types de paysages

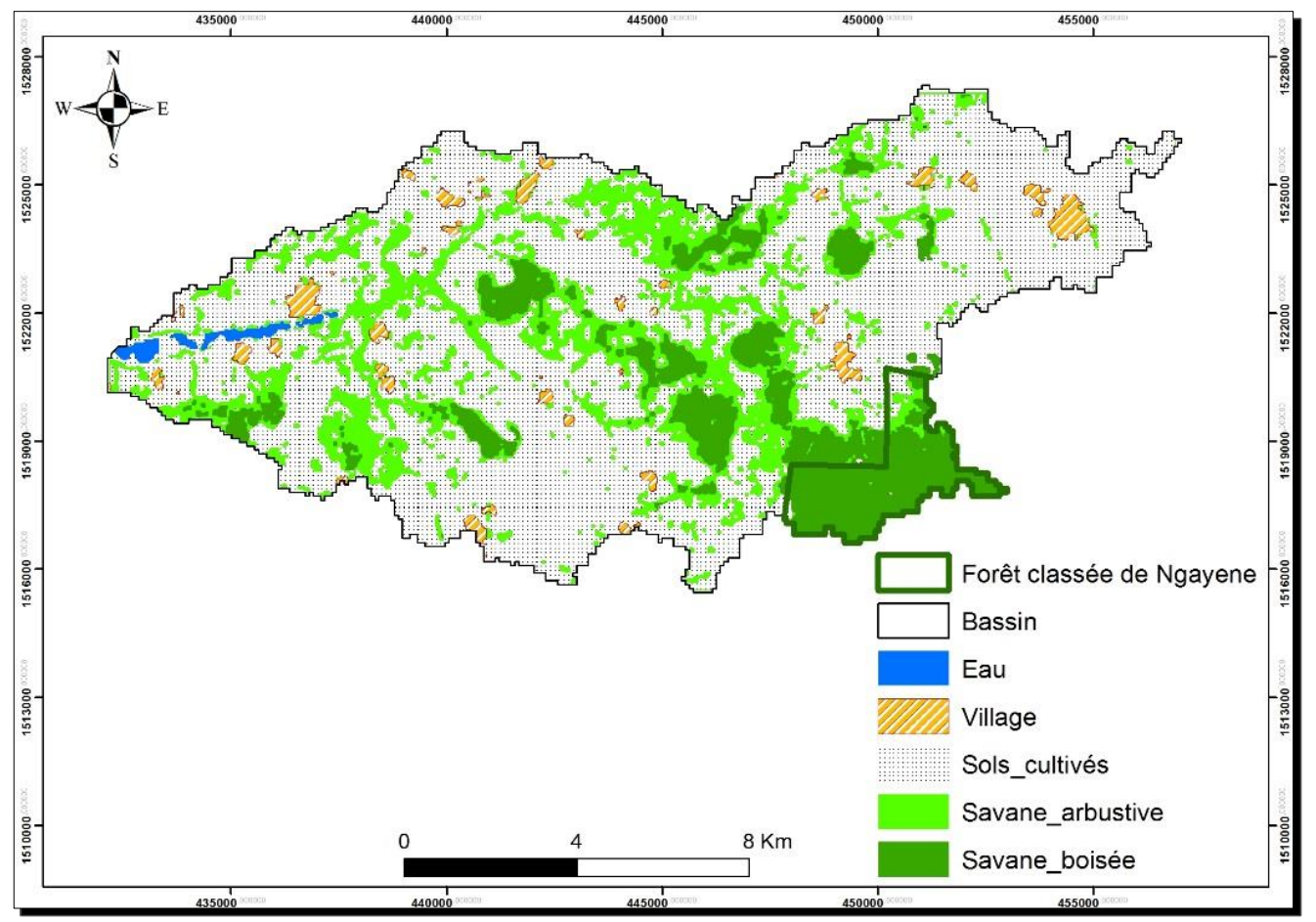

Figure 7 : localisation d'une partie de la forêt classée de Ngayene

L'espace occupé par les eaux en avril est passé de 47 ha en 1996 à 48 ha en 2007 pour être 107 ha en 2016 et cela est la conséquence des aménagements avec notamment la 
construction d'une digue à l'exutoire du bassin et dont le rôle est de stopper l'avancée des eaux salées du baobolong dans vallée de bassin mais également permettre une plus longe conservation des eaux.

\subsection{Sécheresse et hydrologie du bassin de Thysse Kaymore}

L'importance des écoulements sur le bassin de Thysse Kaymore étant intrinsèquement liée à la quantité pluviométrique reçue, la sécheresse qui s'est produite a eu des influences sur l'hydrologie du bassin.

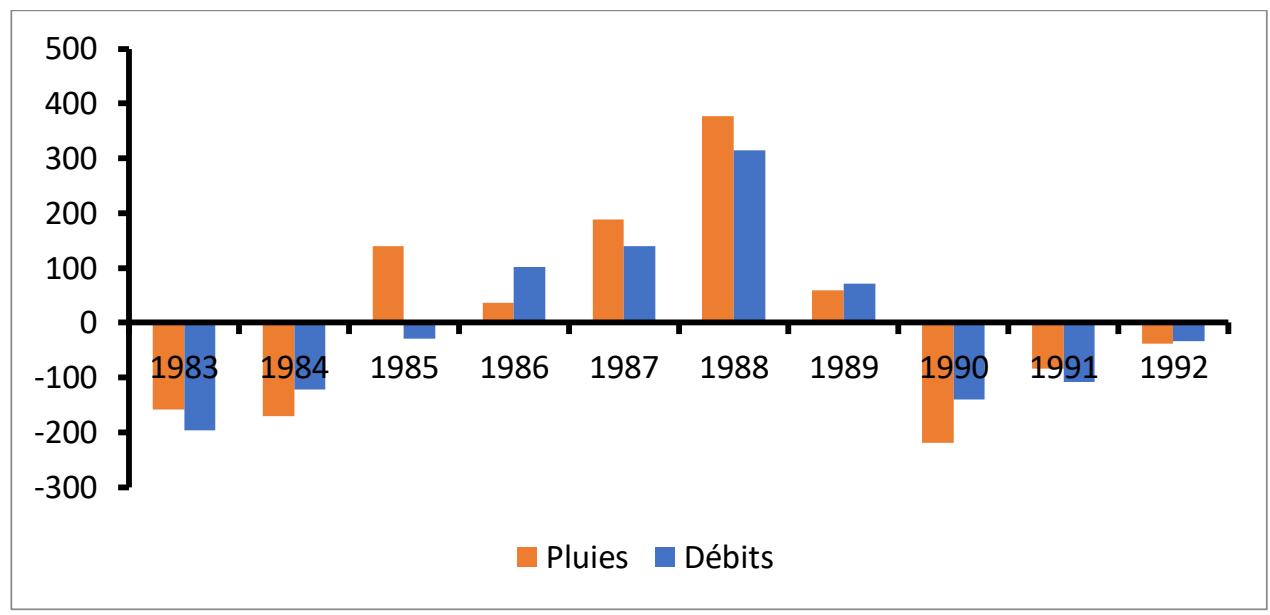

Figure 8 : Relation entre pluviométrie et débits sur le bassin de Thysse

Il existe une relation entre l'évolution pluviométrique et les débits enregistrés sur le bassin de Thysse Kaymore. En effet, sur la période de 1983 à 1992, l'importance des débits enregistrés à la station de Thysse a suivi l'évolution de la pluviométrie. Ce qui fait que sur la période de 1986 à 1989, les débits sont excédentaires par rapport à la moyenne en raison d'une pluviométrie relativement importante (une moyenne de $811 \mathrm{~mm}$ pour cette période). L'année 1988 enregistre le débit le plus élevé avec la pluviométrie la plus importante de la série de donné que nous avons sur Thysse avec $1029 \mathrm{~mm}$. La sécheresse a donc des conséquences sur l'hydrologie du bassin car les années de déficit pluviométrique ont connu des écoulements faibles.

\subsection{Impacts sur les ressources en eau du bassin de Thysse Kaymore}

\subsubsection{Les eaux de surface}

La sécheresse, l'érosion hydrique et le changement de comportement des paysans dans leurs espaces agraires ont des impacts forts sur les ressources en eau de surface du bassin de Thysse de Kaymore. En effet, face à la sécheresse et à dégradation des sols, les bas-fondsfonds sont devenu des zones d'intérêt pour les paysans. Or, la mise en culture de ces unités hydrologiques entraine la destruction de l'équilibre hydrodynamique de ces milieux. Au même moment la dégradation des états de surface des sols entraine une accélération du 
ruissellement occasionnant d'importants ravinements et emportant de fortes quantité de terre estimées à près de 1 tonne/ha/an selon Albergel et al. (1989). Cette situation entraine l'ensablement des bas-fonds qui entraine la réduction de leur profondeur et impacte sur les conditions de stockage et de transfert des eaux. La sécheresse aidant, on note un tarissement précoce des bas-fonds et des mares avec la réduction des totaux pluviométriques annuels et de la durée de l'hivernage.

\subsubsection{Les eaux souterraines}

La principale nappes captées dans la zone est le Continental Terminal (CT) même si le Maestrichtien dont le toit se trouve à la cote -300m (Albergel et al. 1991) couvre la zone. Les quatre forages qui existent dans le bassin de Thysse Kaymore captent tous le C.T à des profondeurs variant entre $79 \mathrm{~m}$ à Thysse Kaymore et $116 \mathrm{~m}$ Keur bakary. Ces forages sont tous fonctionnels et servent à l'alimentation en eau potable des populations.

Tableau 1: Caractéristiques des forages du bassin de Thysse Kaymore

\begin{tabular}{|c|c|c|c|c|c|}
\hline Forage & Aquifère & $\begin{array}{c}\text { Profondeur } \\
\text { Totale }\end{array}$ & $\begin{array}{c}\text { Profondeur } \\
\text { Equipée }\end{array}$ & Rabattement & Date \\
\hline Keur Bakary & C.T & 116 & 107,8 & 3,82 & 1985 \\
\hline Kaymore & C.T & 80 & 63 & 2,76 & 1988 \\
\hline Dimagadio & C.T & 103 & 98 & 54,73 & 2000 \\
\hline Thysse Kaymore & C.T & 79 & 76,4 & 15,78 & 2013 \\
\hline
\end{tabular}

L'importance du rabattement de la nappe du C.T dans le bassin de Thysse Kaymore au niveau des forages de Diamagadio avec $54,73 \mathrm{~cm}$ et Thysse Kaymore 15,78cm indique une baisse du niveau piézométrique même si cette baisse n'est pas uniforme sur l'ensemble du bassin. Le CT est atteint à des profondeurs variant entre $11 \mathrm{~m}$ à côté du grand baobolon et $57 \mathrm{~m}$ sur le plateau par les puits. Cependant, la plupart des puits connaissent des tarissements en saison sèche posant des problèmes d'approvisionnement en eau.

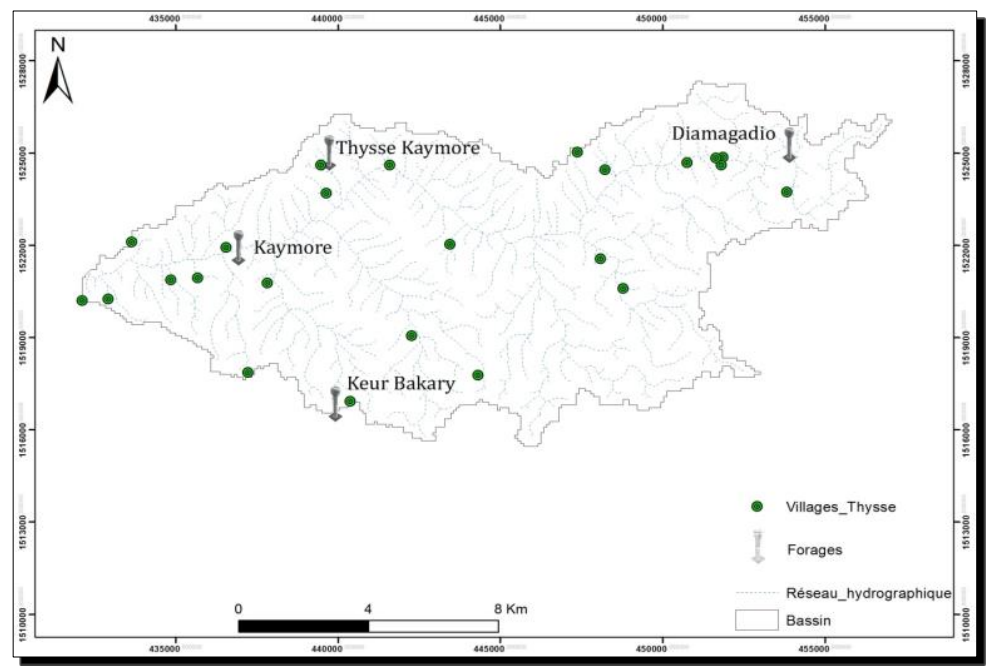

Figure 9: Distribution des ouvrages hydrauliques dans le bassin de Thysse Kaymore 
Pour étudier l'évolution du niveau piézométrique de la zone, quatre puits ont été suivis régulièrement par Albergel et al. (1991) dont une série de mesures réalisées en Juillet, Octobre 1989 et Février 1990 a permis de caractériser les variations piézométriques.

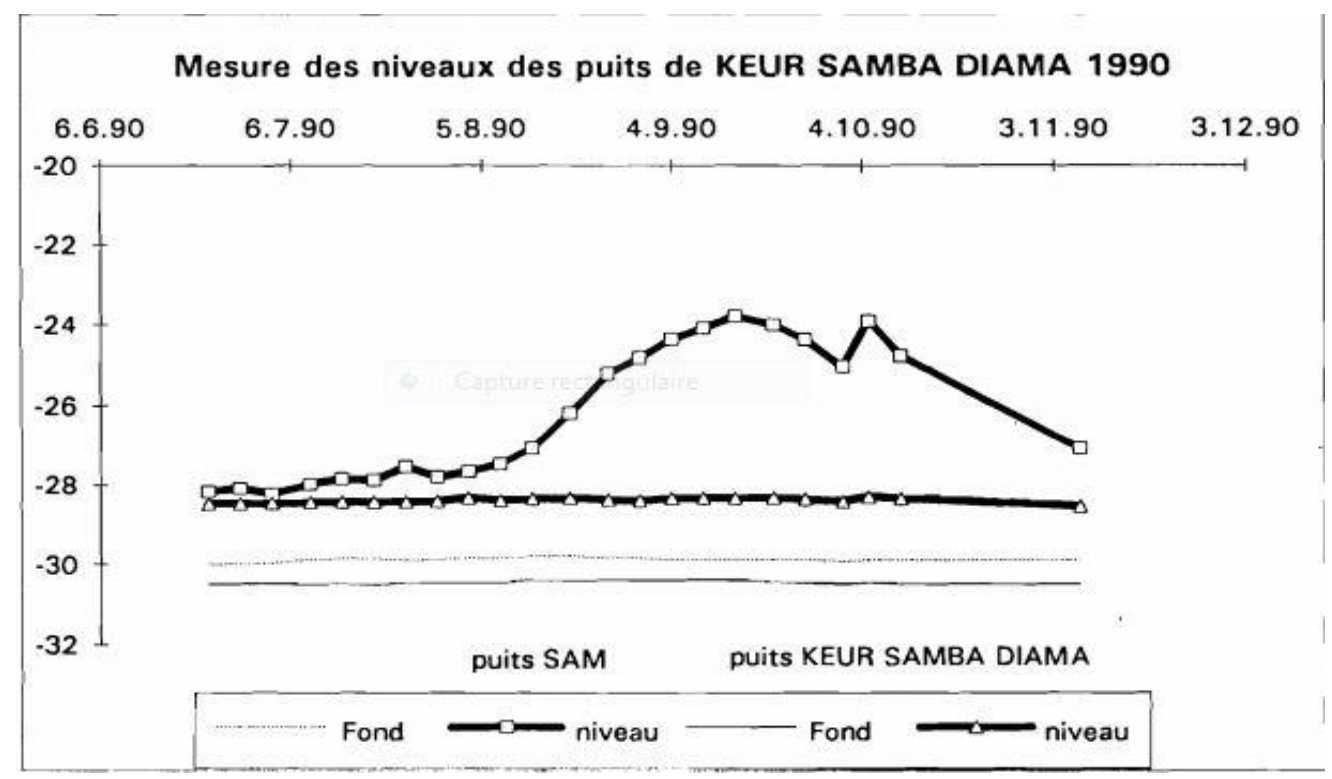

Figure 10: Mesure des niveaux de spuits de Keur Samba Diama 1990 (Dacosta et al., 1992)

Le suivi a permis de constater que les variations sont dans l'ensemble positives durant la saison des pluies et négatives par la suite. Elles peuvent atteindre exceptionnellement plusieurs mètres dans certains Puits (à SAM par exemple) mais elles sont généralement de l'ordre de $20 \mathrm{~cm}$ près des bas-fonds et de quelques centimètres dans les inter-fluves. Cette situation montre que le niveau des nappes monte en hivernage avec la recharge et diminue en saison sèche. La recharge des nappes dépendant de la pluie, la sécheresse a entrainé la baisse du niveau des nappes et par conséquent le tarissement précoce de certains puits en saison sèche. Exemple, à Diamagadio, on comptait sept puits dont un seul ne tarissait pas en saison sèche en 2000 et à Ngayene 64puits dont la plupart tari au maximum de la saison sèche (GERAD, 1996, 2000). Cette baisse du niveau des nappes est confirmée par la DGPRE (2014) qui estime que l'évolution des ressources en eau du bassin arachidier dans lequel se trouve le bassin de Thysse Kaymore, est marquée depuis la fin des années 60 par une baisse continue des nappes phréatiques, un assèchement quasi-permanent des vallées continentales.

\section{Conclusion}

Le bassin versant de Thysse Kaymore connait ces dernières décennies une forte anthropisation qui intervient dans un contexte climatique défavorable marqué par une sécheresse persistante. La réduction des totaux pluviométriques annuels, la forte variabilité interannuelle des précipitations et les mutations des modes d'occupation et d'utilisation de l'espace ont considérablement modifié les états de surface du bassin avec notamment l'extension des cultures à des espaces vulnérables, la disparition de la jachère, la 
surexploitation et la déforestation. La forte pression exercée sur les ressources naturelles du bassin fait qu'aujourd'hui les sols cultivés, les savanes arbustives et les savanes boisées qui représentaient respectivement $31 \%, 27 \%$ et $40 \%$ des superficies du bassin en 1986 sont passées à $62 \%, 21 \%$ et $12 \%$ en 2016 . Consécutivement, la population du bassin basin a doublé en passant de 10679hbts en 1988 à 23140 hbts en 2013. Cette situation a entrainé une forte dégradation des ressources en eau du bassin de Thysse Kaymore avec une intensification du ruissellement, l'ensablement des bas-fonds et la modification des conditions de stockages de d'eau. Cette situation conjuguée aux déficits pluviométriques chroniques et à l'augmentation de la pression démographique ont provoqué une baisse continue du niveau des nappes. Ainsi, le tarissement précoce des mares d'eau et de certains puits rendent difficile les conditions d'accès à l'eau pour les populations mais également pour le cheptel.

La digue construite à l'exutoire du bassin récemment devait permettre un plus grand stockage de l'eau qui se perdait dans le baobolong et permettre ainsi un meilleur accès à l'eau pour le maraîchage qui se développe dans cette zone, mais également pour le bétail. Mais cette action devait s'accompagner de l'aménagement des bas-fonds par la mise en défend pour permettre de ralentir le ruissellement, d'endiguer l'érosion et favoriser l'infiltration pour la recharge des nappes.

\section{Références bibliographiques}

1. Albergel J. et al., 1989. Méthode pour améliorer l'infiltration et réduire le ruissellement, présentation du cas du Sine-Saloum (Sénégal) ORSTOM, ISRA, IRAT, ISRA/SCS- Recherches d'appui Sine-Saloum-Septembre 1989, 18p.

2. Albergel J.et al.,1989. Rapport hydrologique 1989 bassin versant de Thysse Kaymor, Action de recherche "DRS-Economie de l'eau », Programme CEE-CIRAD N TS2A 0017 F CD, 51p.

3. Albergel J. et al., 1991. Projet Pilote " Sine Saloum " Bas-fond de THYSSE KAYMOR Rapport de synthèse : HYDROLOGIE- HYDROGEOLOGIE, 33p

4. Albergel J. et al., 1995. Réhabilitation d'un écosystème semi-aride au Sénégal par l'aménagement des éléments du paysage in L'homme peut-il refaire ce qu'il a défait? R. Pontanier, A. M'Hiri, N. Akrimi, J. Aronson, E. , 293-306,LeFloc'h John Libbey Eurotext, Paris

5. Albergel J. et al.,2000. Aménagement hydraulique et bocage dans le bassin arachidier du Sénégal in La jachère en Afrique tropicale-CH. Floret, R. Pontanier, John Libbey Eurotext, Paris 2000, PP. 741-750.

6. Dacosta H., Kandia K.Y., Malou R., 2002. La variabilité spatio-temporelle des précipitations au Sénégal depuis un siècle. in FRIEND 2002: Regional hydrology: bredging the gap between Research and Practice (Proccedings of the Fourth international FRIEND conference held at Cap Town, South Africa, March 2002), IAHS Publ., 274 : 499-506.

7. Descroix L. et al., 2009. Spatio-temporalvariability of hydrological regimes around the boundaries between Sahelian and Sudanian areas of West Africa: a synthesis. J. Hydrology, 375, 90-102.

8. Diallo M. A., 1995. Evolution de la pluviométrie au Sahel, Internal Repport, Inetrnational Atomic Energy Agency and United Nations Educational Scientific and cultural organisation, Inetrnational centre for theorical physic, $6 \mathrm{p}$. 
9. Diatta M., 1994. Mise en défens et techniques agroforestières au Sine Saloum (Sénégal). Effets sur la conservation de l'eau et du sol et sur la production primaire, Thèse de doctorat de l'Université Scientifique L. Pasteru (Strasbourg I), 199p

10. DGPRE, 2014. Plan d'action de gestion intégrée des ressources en eau (PAGIRE) projet de mise en cuvre du PAGIRE dans le bassin arachidier (PAGIRE-BA) étude d'évaluation et de suivi des ressources en eau de surface, $R 3$-rapport sur la modélisation des ressources en eau et les normes de conception des ouvrages.

11. Faye E. et al., 2000. Le bois et la gestion des jachères soudaniennes du bassin arachidier au Sénégal in La jachère en Afrique tropicale-Ch. Floret, R. Pontanier, John Libbey Eurotext, Paris, 2000, PP. 451-459.

12. ISRA, 2010. Zone du bassin arachidier, détail publié le mercredi 2juin 2010 disponible sur www.isra.sn/index.php/component/content/article/88.html.

13. Lericolais A., 1972. SOB étude géographique d'un terroir Sérèr (Sénégal), Atlas des structures agraires au sud du Sahara. 7, collection publiée sous le patronage de la maison des sciences de l'Homme, Paris, MOUTON \& CO LA HAYE, 110p.

14. Lericolais A., 1999. Paysans Sereer Dynamiques agraires et mobilités au Sénégal

15. Lienou G. et al., 1999. Caractéristiques des relations eaux de surface-eaux souterraines en milieu tropical sec : exemple du bassin de la Nema (Sine-Saloum, Sénégal). Sud Sciences Technologies, 3, 39-46

16. Mahé J. et al., 2013. The rivers of Africa: witness of climate change and human impact on the environment, Hydrological Processes, 27, 2106-2114.

17. Minea I., Stângă I.C., 2004. Analiza variabilităţii spaţiale a unor indici de apreciere a secetelor, Riscuri şi Catastrofe III, Editor Victor Sorocovschi, Edit. Casa Cărţii de Ştiinţă, pag. 138-149, ISSN 1584-5273, Cluj-Napoca.

18. Ndong J.B., 1995. L'évolution de la pluviométrie au Sénégal et les incidences de la sécheresse récente sur l'environnement / The evolution of rainfall in Senegal and the consequences of the recent drought on the environment. Revue de géographie de Lyon, 70(3-4), 193-198

19. Perez P., 1994. Genèse du ruissellement sur les sols cultivés du sud-Saloum (Sénégal) du Diagnostic à l'aménagement de parcelle, Thèse doctorat, Ecole Nationale Supérieure Agronomique de Montpellier.

20. République du Sénégal, Direction de l'Analyse et de la Prévision des Statistiques (DAPS), 2009. Rapport de l'étude sur l'évolution du secteur agricole, des conditions de vie des ménages et de la vie chère au Sénégal.

21. République du Sénégal, Ministère du réseau hydrographique national, GCO/SEN, 2007. Programme national d'appui à la sécurité alimentaire, projet de petite hydraulique villageoise dans le bassin arachidier, Rapport de formulation.

22. Sagna P.,1995. L'évolution pluviométrique récente de la Grande-Côte du Sénégal et de l'archipel du Cap-Vert, article, Revue de géographie de Lyon, Année 1995, Volume 70, Numéro 3, pp. 187-192 
